\title{
Antiferroelectric dielectric relaxation processes and the interlayer interaction in antiferroelectric liquid crystals
}

\author{
Jang-Kun Song, U. Manna, A. Fukuda, and J. K. Vij ${ }^{\text {a) }}$ \\ Department of Electronic and Electrical Engineering, Trinity College, University of Dublin, \\ Dublin 2, Ireland
}

(Received 18 June 2008; accepted 8 August 2008; published online 7 October 2008)

\begin{abstract}
Antiphase relaxation process of antiferroelectric phase is investigated in binary mixtures of antiferroelectric and ferroelectric liquid crystalline compounds. It is found that the frequency of the antiphase mode ranges from 2 to $4 \mathrm{MHz}$, being one of the fastest collective processes reported so far. This can be exploited in a photonic switch with a submicrosecond response time. However, the frequency of the antiphase mode decreases by the mixing ratio of the ferroelectric compound. The decrease in the frequency is shown to be due to a reduction in the elastic constant in between the adjacent layers. (0) 2008 American Institute of Physics. [DOI: 10.1063/1.2977871]
\end{abstract}

The frequency dependence of the dielectric permittivity of liquid crystal cells reflects the molecular motions induced by applied fields. A number of dielectric relaxation processes have been found in liquid crystal cells and these can be broadly classified as the following: molecular and collective processes. ${ }^{1-3}$ While the molecular processes correspond to the independent motions of individual molecules, where the macroscopic change in the average alignment of molecules is not detected, the collective processes arise from the collective motion of molecules. The latter are directly related to electro-optical switching properties of the liquid crystal cells. Usually, the molecular processes appear at higher frequencies than do the collective ones implying that the individual molecular motions are faster than the collective ones. However, this is not always the case. The fastest collective mode is found in the antiferroelectric phase. Hiraoka et al. reported that the antiphase process, collective mode as explained schematically in Fig. 1(a), is faster than the reorientation around the molecular short axis in MHPOBC. ${ }^{4}$ The relaxation frequency was reported to reach up to $0.9 \mathrm{MHz}$ at a higher temperature, though it is less than $10 \mathrm{kHz}$ at lower temperatures. Marzec et al. recently found that the frequency of an antiphase process varies from $\sim 0.65$ to $2.9 \mathrm{MHz}$ for $3 \mathrm{~F} 4 \mathrm{Bi}$ and from $\sim 1.0$ to $2.4 \mathrm{MHz}$ for $1 \mathrm{~F}(2 \mathrm{H}) 3 \mathrm{Bi}^{5}{ }^{5}$ Interestingly these compounds also exhibit the same phase sequence as reported here. It is therefore desirable to further investigate the high frequency collective modes, since these can predict limitations of the response time of the material for various applications such as in liquid crystal displays. Note that the conventional liquid crystal displays still suffer from the slow response time. There exist two collective modes in the chiral smectic liquid crystals: the Goldstone mode associated with the fluctuation of the azimuthal angle $(\phi)$ around the helical axis and the soft mode corresponding to the fluctuation of the molecular tilt angle $(\theta) .{ }^{1-3}$ However, this definition becomes inadequate in the antiferroelectric phase, since the two types of fluctuations of the azimuthal angle $(\phi)$ may exist in this phase. One of these is the antiferroelectric Goldstone mode. This is similar to the Goldstone mode in the $\mathrm{SmC}^{*}$ phase and represents the unwinding of the long helical structure of the $\mathrm{SmC}_{A}^{*}$ phase, as explained in Fig. 1(b). The other consists of the opposite motions of directors

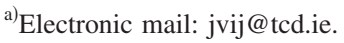

in two adjacent layers, as shown in Fig. 1(a), and was named the antiphase motion by Panarin et al. ${ }^{6}$ and Buivydas et al. ${ }^{7}$ This mode can cause the field induced phase transition from the antiferroelectric to the ferroelectric state and accounts for the dynamics of the pre-transition phenomenon. So, it is misleading to designate it as a Goldstone mode as Hiraoka et al. had first suggested. ${ }^{4}$ Usually, the antiphase mode appears in the frequency range to $10 \mathrm{kHz}-2 \mathrm{MHz}$, which may be one of the fastest collective modes. ${ }^{4,5,7}$

Here, we report that an antiferroelectric liquid crystal, MC881 shows a very high relaxation frequency antiphase mode. We also show that the relaxation frequency of the antiphase motion is decreased by mixing a ferroelectric liquid crystalline compound with MC881. Antiferroelectric liquid crystalline MC881 and ferroelectric liquid crystalline compounds MC815 (Mitsubishi Gas Chemical Company, Inc.), were investigated using dielectric spectroscopy. ${ }^{8}$ The chemical structures of MC881 and MC815 shown in Fig. 2 are quite similar to each other. The tilt angle and the spontaneous polarization of the two compounds do not differ much either, as shown in Fig. 2. However, the phase sequence of $\mathrm{MC} 881$ is $\mathrm{Sm}_{A}^{*}\left(112.5^{\circ} \mathrm{C}\right) \mathrm{Sm}^{*}\left(120^{\circ} \mathrm{C}\right)$ isotropic $(I)$ phase, while that of $\mathrm{MC} 815$ is $\mathrm{SmC}^{*}\left(102^{\circ} \mathrm{C}\right) I$. Thus it is reasonable to designate MC881 and MC815 as antiferroelectric and ferroelectric liquid crystalline compounds, respectively. The dielectric permittivity of MC881 was investigated in both the homeotropic and planar cells using broadband high resolution dielectric spectrometer (Novocontrol GmbH, Germany). Dielectric/optical cells each of $20 \mu \mathrm{m}$ thickness
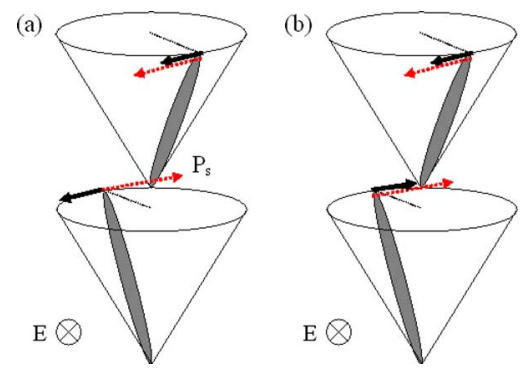

FIG. 1. (Color online) Schematics of the antiphase (a), and the antiferroelectric Goldstone (b) modes in the relaxation processes of an antiferroelectric phase. The red dotted and black solid arrows indicate the spontaneous polarization $\left(P_{s}\right)$ and the direction of molecular motions, respectively. This $\mathbf{c}$ directors shown by narrow lines lie in the smectic layer plane. 


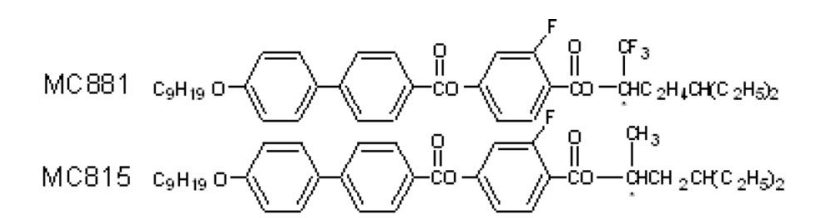

(a)

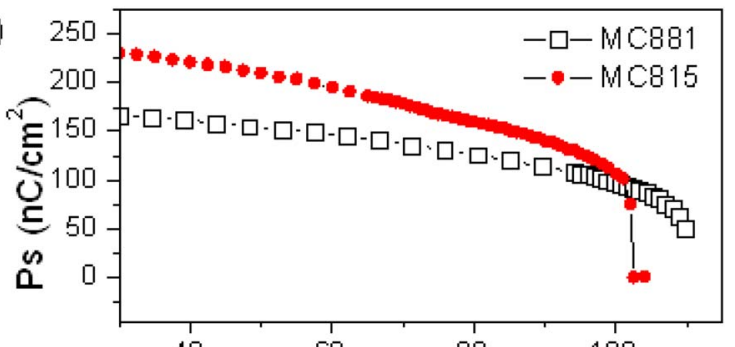

(b)

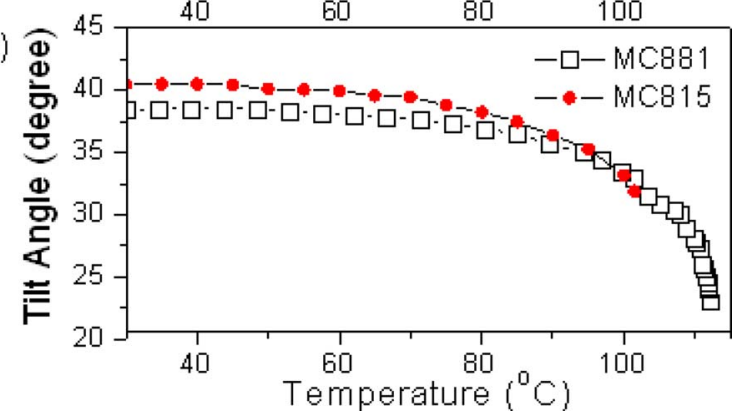

FIG. 2. (Color online) Chemical structure of MC881 (antiferroelectric liquid crystal) and MC815 (ferroelectric liquid crystal), and the spontaneous polarization (a), and tilt angle (b) as function of temperature.

were prepared by using two low resistance indium tin oxide (ITO) glass plates $(20 \Omega / \square)$ coated with an alignment layer. RN-1175 (Nissan Chemical Co.) and Dow Corning 9-6346 Silane [72\% 3-(trimethoxysilyl) propyldimethyloctadecylammonium-chloride and $28 \%$ $\mathrm{MeOH}]$ were used to obtain planar and homeotropic alignments of cells, respectively. Experiments are performed during cooling of the cells from 120 to $80^{\circ} \mathrm{C}$. Mixtures of MC881 and MC815 are also investigated using the same procedure.

To extract the parameters characterizing the relaxation processes, the imaginary part $\left(\epsilon^{\prime \prime}\right)$ of the dielectric permittivity is fitted to Harvriliak-Negami (HN) equation. Figures 3(a) and 3(b) show the plots of the maximum relaxation frequency calculated from the HN frequency and the dielectric strength $\Delta \epsilon$ as a function of temperature for planar and homeotropic cells. From frequencies of $10 \mathrm{kHz}$ to $10 \mathrm{MHz}$, two relaxation processes are found in the planar cell in Fig. 3 . It was reported that the four different processes are possible in the antiferroelectric phase. ${ }^{6,9}$ The lowest frequency process is the helical unwinding process or the antiferroelectric Goldstone process, which appears at frequencies of less than $1 \mathrm{kHz}$ in MC881, and is overlapped by the conductivity mode due to the ionic impurities and hence it is not easy to separate these two modes. Hence, Fig. 3 does not include the antiferroelectric Goldstone mode. The other three processes are the molecular relaxation processes: rotation around the molecular short axis (which appears in the lower frequency range) and the relaxation around the molecular long axis (highest frequency process $(\approx 1 \mathrm{GHz})$, beyond our experimental window), and the antiphase process induced by the oppositely rotating motions of the $\mathbf{c}$ directors in the neighboring layers. Therefore, the two processes observed in the planar cell in Fig. 3 are the modes for the low frequency

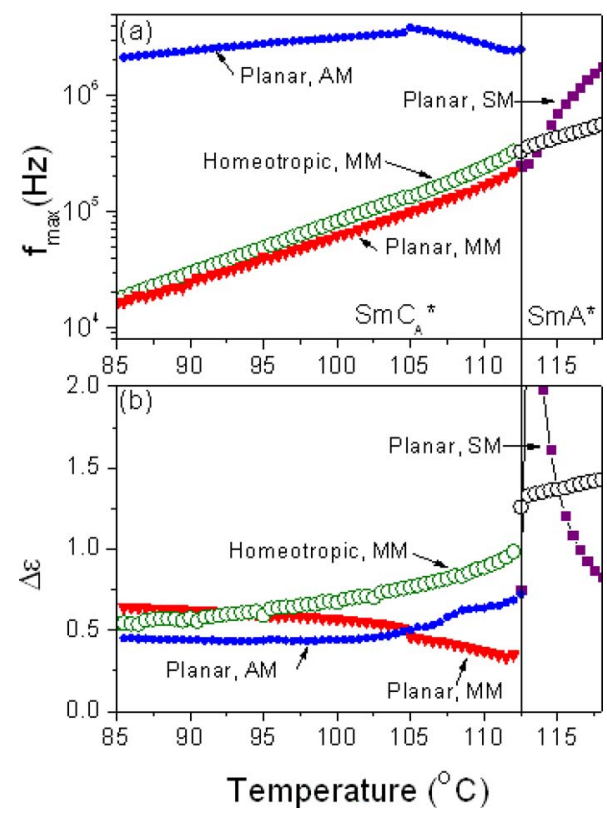

FIG. 3. (Color online) The relaxation frequency (a) and the dielectric strength $\Delta \epsilon(\mathrm{b})$ of pure MC881 as function of temperature. MM, AM, and SM denote the molecular, antiphase and soft modes respectively.

molecular relaxation and the antiphase processes, respectively. As an aid for identifying the two relaxation processes of the planar cell, we compare the results of the planar and homeotropic cells. In Fig. 3(a), the homeotropic cell shows a single relaxation process, which is continuous from $\mathrm{Sm} A^{*}$ to $\mathrm{SmC}_{A}^{*}$ phases. This implies that the relaxation process in the homeotropic cell is the molecular rotational process along the molecular short axis. Note that the antiferroelectric Goldstone and the antiphase modes cannot appear in the homeotropic cell, because the field direction is perpendicular to the smectic layers, and the applied field cannot induce any azimuthal motion in the directors. The molecular process observed in the homeotropic cell has almost the same frequency as the low frequency process observed in the planar cell. This means that this low frequency relaxation in the planar cell is the molecular process. Therefore, the remaining high frequency process in the planar cell must be the antiphase process. ${ }^{6,7}$ As shown in Fig. 3(a), the frequency of the antiphase mode lies in the range of $2-4 \mathrm{MHz}$, which as far as we are aware is one of the fastest such collective processes reported to date. ${ }^{4,5}$ Moreover, the frequency of the antiphase mode is faster than that of the soft mode in $\mathrm{Sm} A^{*}$ phase [Fig. 3(a)].

It is also interesting to investigate the behavior of the mixtures of MC881 and MC 815 . We added $12 \%$ and $20 \%$ by weight of MC815 into MC881 to make two mixtures of $88 \%$ and $80 \%$ MC881. By adding MC815 into MC881, SmC* phase appears in the temperature range between $\operatorname{Sm} A$ and $\mathrm{SmC}_{A}^{*}$ phases. The phase sequence of the $88 \%$ MC881 mixture is $\operatorname{Sm}_{A}^{*}\left(110^{\circ} \mathrm{C}\right) \mathrm{Sm}^{*}\left(114^{\circ} \mathrm{C}\right) \mathrm{Sm}^{*}\left(120^{\circ} \mathrm{C}\right) \mathrm{I}$, and that of $80 \% \mathrm{MC} 881$ mixture is $\operatorname{SmC}_{A}^{*}\left(105^{\circ} \mathrm{C}\right) \mathrm{SmC}^{*}$ $\left(112{ }^{\circ} \mathrm{C}\right) \mathrm{Sm}^{*}\left(120^{\circ} \mathrm{C}\right) \mathrm{I}$. Figure 4 shows the relaxation frequency of the antiphase and the molecular modes in the antiferroelectric phase of the mixtures. The frequency of the molecular mode is not affected by mixing of the two liquid crystalline compounds [Fig. 4(b)], whereas the frequency of the antiphase mode does vary with the mixing ratio [Fig. 4(a)]. An examination of the difference in the origins of the 


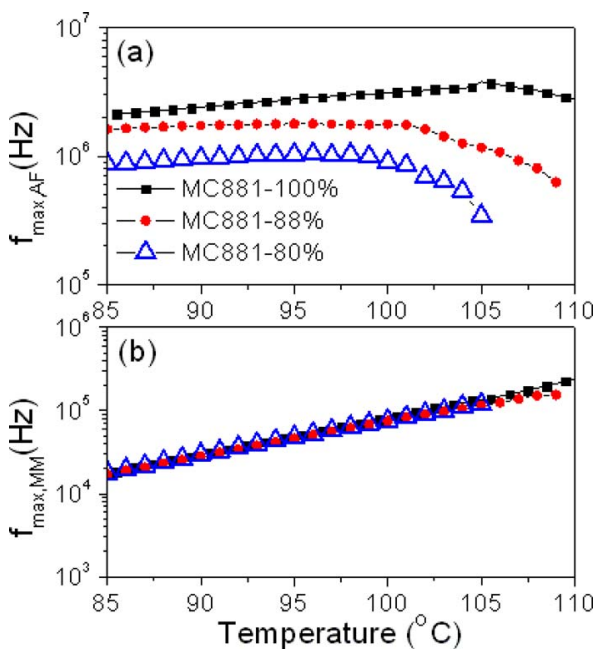

FIG. 4. (Color online) Relaxation frequencies of the antiphase (AF) and molecular modes (MM) versus temperature for different mixing ratios.

two relaxation processes allows one to deduce the difference in the dependencies of the relaxation frequency on the mixing ratio. The molecular mode arises from the individual molecular fluctuations, hence it is related to the intermolecular interactions between the molecules. However, the antiphase mode resulting from the motion of molecules in the adjacent smectic layers is related to the interlayer interactions between the neighboring layers. As explained in the previous section, MC815 is a ferroelectric liquid crystal compound having quite a similar chemical structure to MC881. A negligible dependence of the relaxation frequency of the molecular mode on the mixing ratio may be due to the similar chemical structures of the two liquid crystalline components, that is, strength of the intermolecular interactions may not vary very much by mixing a small amount of MC815 into MC881. This rapid fall in the frequency of the antiphase mode with the addition of the ferroelectric liquid crystalline compound implies that the interlayer interaction depends rather sensitively on the mixing ratio. Though a more accurate calculation may be required in obtaining an exact expression for the frequency of the antiphase mode, we can intuitively estimate that the frequency of the antiphase mode ${ }^{6}$ is proportional to the elastic constant $(K)$ between the two adjacent layers. $f_{\max } \approx K / \gamma$, where $\gamma$ is the rotational viscosity between the layers.

According to the phenomenological theory of the antiferroelectric phases, ${ }^{10,11}$ the interlayer interaction between the two adjacent layers has both dipolar and quadrupolar terms. This is now well confirmed experimentally. ${ }^{12,13} \mathrm{By}$ ignoring the term due to flexoelectric polarization, which may be quite small in spite of the essential role it does play in the evolution of subphases, ${ }^{11}$ and additionally by ignoring the tilt angle dependent term based on a constant tilt angle approximation, we can simplify the free energy in terms of the azimuthal angle $(\phi)$ in between the c directors of the two neighboring layers as $F$ $=\sum\left(-a\left(T-T_{0}\right) \cos \phi_{i, i+1}-B \cos ^{2} \phi_{i, i+1}\right)$, where the positive coefficient $a$ represents the antiferroelectric interaction and positive coefficient $B$ makes the molecules in the two adjacent layers placed in one plane, and $T_{0}$ is the transition temperature from $\mathrm{SmC}_{A}^{*}$ to $\mathrm{Sm} C^{*}$. In the $\mathrm{SmC}_{A}^{*}$ phase, $\Delta T \equiv(T$ $\left.-T_{0}\right)$ is negative so that anticlinic ordering is more stable. Larger $a$ means a more stable antiferroelectric state. The elastic constant between the layers can be defined as $K$ $\equiv \partial^{2} F / \partial \phi_{i, i+1}^{2}\left(\phi_{i, i+1}=\pi+\delta \phi\right)$ at the anticlinic order. Therefore, we find the elastic constant between the layers as $K$ $=a\left(T_{0}-T\right)+2 B$. Thus $K$ is proportional to the antiferroelectric coefficient $a$, which means that strong antiferroelectric interaction will increase the elastic constant for the anticlinic ordering. It is reasonable to assume that the antiferroelectric coefficient $a$ of an antiferroelectric liquid crystal may decrease upon mixing with a ferroelectric liquid crystalline compound. Therefore, we can deduce that the elastic constant decreases by increasing the percentage of the added ferroelectric liquid crystal, which causes a decrease in the relaxation frequency of the antiphase mode.

We measured the frequency dependence of dielectric permittivity of MC881 and MC815, antiferroelectric and ferroelectric liquid crystal mixture systems. Pure MC881 exhibits significantly large values of the relaxation frequency of the antiphase mode, in the frequency range of $2-4 \mathrm{MHz}$, the fastest collective processes reported so far. Such a large frequency can be exploited in a photonic switch with a submicrosec response time, which might be the fastest liquid crystalline device known so far. The relaxation frequency decreases by increasing the ratio of MC815 in the mixtures of MC881 and MC815. By adding a ferroelectric liquid crystalline compound, the antiferroelectric coefficient $a$ in the Landau free energy expression of antiferroelectric phase decreases and hence the elastic constant in the anticlinic order decreases too. We suggest that a decrease in the elastic constant caused by mixing a ferroelectric liquid crystalline compound is the origin of the phenomenon whereby the relaxation frequency of the antiphase mode decreases by increasing the ferroelectric component in the mixture. This explains as to why the frequency of the antiphase mode of MC881 is the highest amongst the data reported so far; MC881 is a pure antiferroelectric material not showing $\mathrm{SmC}^{*}$ phase, while the other materials reported so far such as MHPOBC and $\mathrm{MHP}(3 \mathrm{H}) \mathrm{BBC}$ have both $\mathrm{SmC}^{*}$ to $\mathrm{SmC}_{A}^{*}$ direct transitions. Therefore, the interlayer interaction responsible for antiferroelectricity in MC881 is supposed to be possibly the largest.

The authors thank SFI for funding (02/IN.1/I.031 and RFP06/ENE039) and Mitsubishi Gas Chemical, Co., Japan for donating their valuable compounds.

${ }^{1}$ A. Levstik, T. Carlsson, C. Filipic, I. Levstik, and B. Zeks, Phys. Rev. A 35, 3527 (1987).

${ }^{2}$ A. M. Biradar, S. Wrobel, and W. Haase, Phys. Rev. A 39, 2693 (1989).

${ }^{3}$ F. Gouda, K. Skarp, and S. T. Lagerwall, Ferroelectrics 113, 165 (1991).

${ }^{4}$ K. Hiraoka, H. Takezoe, and A. Fukuda, Ferroelectrics 147, 13 (1993).

${ }^{5}$ M. Marzec, A. Mikulko, S. Wrobel, and W. Haase, Dielectric Properties of Liquid Crystals, edited by Z. Galewski and L. Sobczyk (Transworld Research Network, Kerala, India, 2007), pp. 83-101.

${ }^{6}$ Yu. P. Panarin, O. Kalinovskaya, and J. K. Vij, Appl. Phys. Lett. 72, 1667 (1998); Yu. P. Panarin, O. Kalinovskaya, and J. K. Vij, Liq. Cryst. 25, 241 (1998).

${ }^{7}$ M. Buivydas, F. Gouda, S. T. Lagerwall, and B. Stebler, Liq. Cryst. 18, 879 (1995).

${ }^{8}$ J. K. Song, U. Manna, A. Fukuda, and J. K. Vij, Appl. Phys. Lett. 91, 042907 (2007).

${ }^{9}$ Yu. P. Panarin, O. Kalinovskaya, J. K. Vij, and J. W. Goodby, Phys. Rev. E 55, 4345 (1997).

${ }^{10}$ H. Orihara and Y. Ishibashi, Jpn. J. Appl. Phys., Part 2 29, L115 (1990).

${ }^{11}$ A. V. Emelyanenko and M. A. Osipov, Phys. Rev. E 68, 051703 (2003).

${ }^{12}$ T. Qian and P. L. Taylor, Phys. Rev. E 60, 2978 (1999).

${ }^{13}$ J. K. Song, A. Fukuda, and J. K. Vij, Phys. Rev. E 76, 011708 (2007) 